\title{
Liquid Stage in-situ Growth of Pd Nanoparticles in a Surfactant Liquid Crystal Template
}

\author{
Lucas R. Parent ${ }^{1}$, David B. Robinson ${ }^{2}$, James E. Evans ${ }^{3}$, and Ilke Arslan ${ }^{1}$ \\ ${ }^{1}$ Department of Chemical Engineering and Materials Science, University of California-Davis, One \\ Shields Ave., Davis, CA 95616 \\ ${ }^{2}$ Sandia National Laboratories, 7011 East Ave. Livermore, CA 94550 \\ ${ }^{3}$ Department of Molecular and Cellular Biology, University of California-Davis, One Shields Ave., \\ Davis CA 95616
}

As the global demand and consumption of clean and renewable electrical energy continues to grow, there is a complementary need for efficient energy storage technologies that charge rapidly. Currently, mesoporous metal nanoparticles, particularly Pd, are being examined for potential uses in hydrogen storage, electrochemistry (double-layer capacitors), and catalysis [1]. In all these applications, surface interactions control performance. An ideal structure would have a highlyconnected pore morphology, where any point in the structure is within a few atoms of a surface interface. Such a structure would exhibit rapid mass transport to the pore surface, faster reaction kinetics within the structure, and high capacitance in electrochemical double-layer capacitors. Palladium nanoparticles have been grown with interconnected $\sim 3 \mathrm{~nm}$ pores using a metal-surfactant paste, and it has been shown that the pore structure and morphology can be controlled by synthesis conditions $[2,3]$. In order to synthesize porous $\mathrm{Pd}$ nanostructures with optimal pore morphology for hydrogen storage, the growth of Pd in the surfactant network must first be understood.

Using in-situ STEM microscopy and a fluid stage by Hummingbird Scientific, the e-beam growth of Pd nanostructures can be recorded with nanometer spatial resolution. The in-situ fluid stage consists of 2 silicon nitride windows, each $50 \mathrm{~nm}$ thick. The chips are stacked, being separated by 50-70nm tall gold spacers, providing a channel for the liquid ( $\mathrm{Pd}$ paste). Here we us this in-situ experimental setup on aqueous Pd salt solutions (control) and Pd-surfactant pastes. Figure 1 shows the progressive growth of $\mathrm{Pd}$ nanostructures from the Pd salt solution. Each image is taken $\sim 10$ seconds after the preceding image, providing an indication of the growth rate. The Pd growth consists of initial homogeneous nucleation of seed particles, followed by the coarsening and fusing of the small particles to grow large networks. Figure 2 shows the growth of Pd structure within the surfactant support. The initial nucleation of seed particles was not captured, but these images show the agglomeration and fusing of small particles, forming a larger porous Pd network. Diffusion and growth kinetics of $\mathrm{Pd}$ in the surfactant paste is much slower than in the aqueous solution. Far less nucleation of seed Pd particles occurs in the surfactant paste. Instead, growth of large networks is more dependent on the motion and agglomeration of existing Pd particles.

[1] F. A. Lewis, International Journal of Hydrogen Energy 21 (1996) 461.

[2] D. B. Robinson, S. J. Fares, M. D. Ong, I. Arslan, M. E, Langhan, K. L. Tran, W. M. Clift, International Journal of Hydrogen Energy 34 (2009) 5585-5591.

[3] D. B. Robinson, S. J. Fares, M. D. Ong, M. E, Langhan, B. W. Woods, W. M. Clift, L. K. Murton, R. P. Hjelm, M. S. Kent, International Journal of Hydrogen Energy 35 (2010) 54823-5433. 


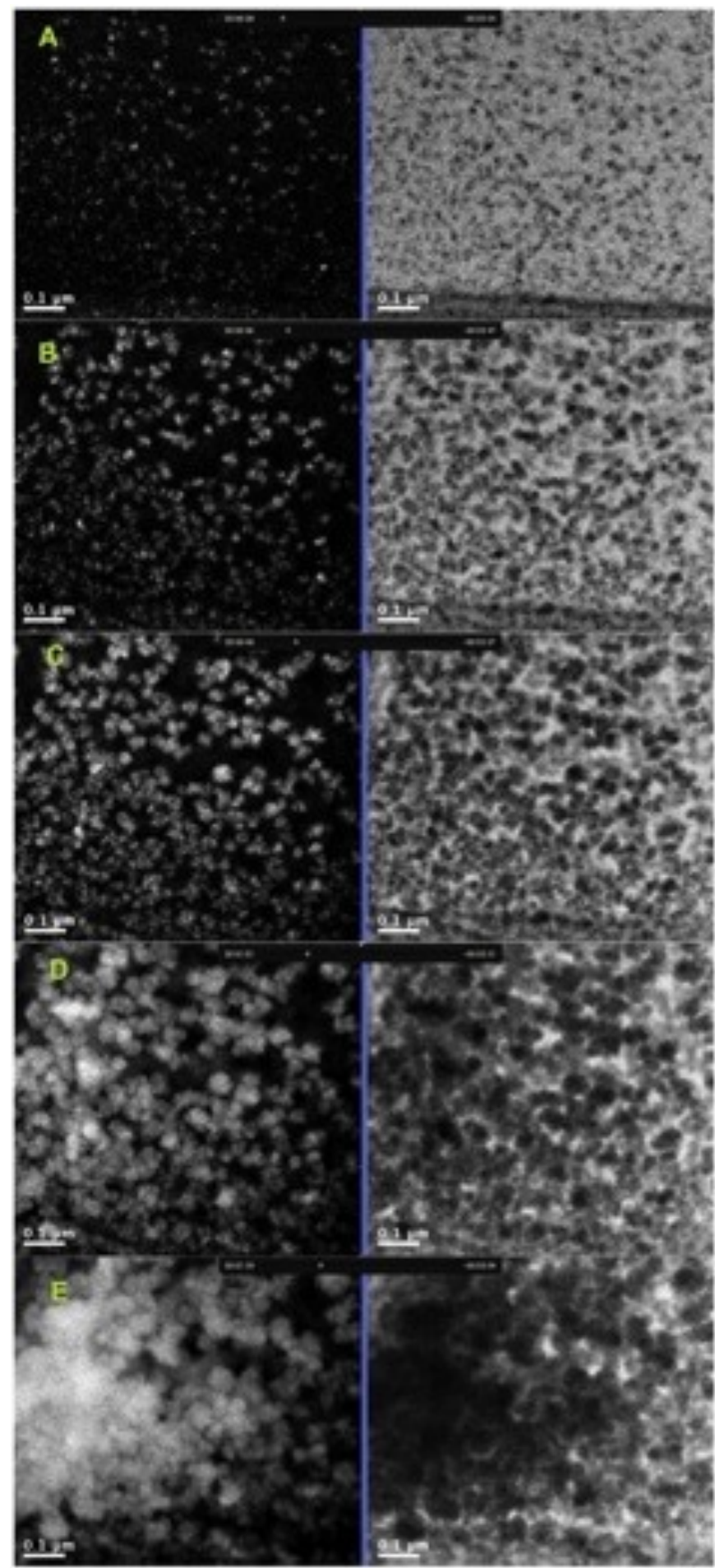

Figure 1: STEM images (DF image left, BF image right, mag x200k) over time during the e-beam growth of Pd particles from a Pd salt solution (no surfactant). Each image is 10 seconds after the preceding image. Image A shows the initial homogenous nucleation of seed particles. In $\mathrm{B}$ the heterogeneous coarsening begins, which continues through $\mathrm{C}, \mathrm{D}$, and $\mathrm{E}$, eventually creating a large $(\sim 1 \mu \mathrm{m})$ Pd network.

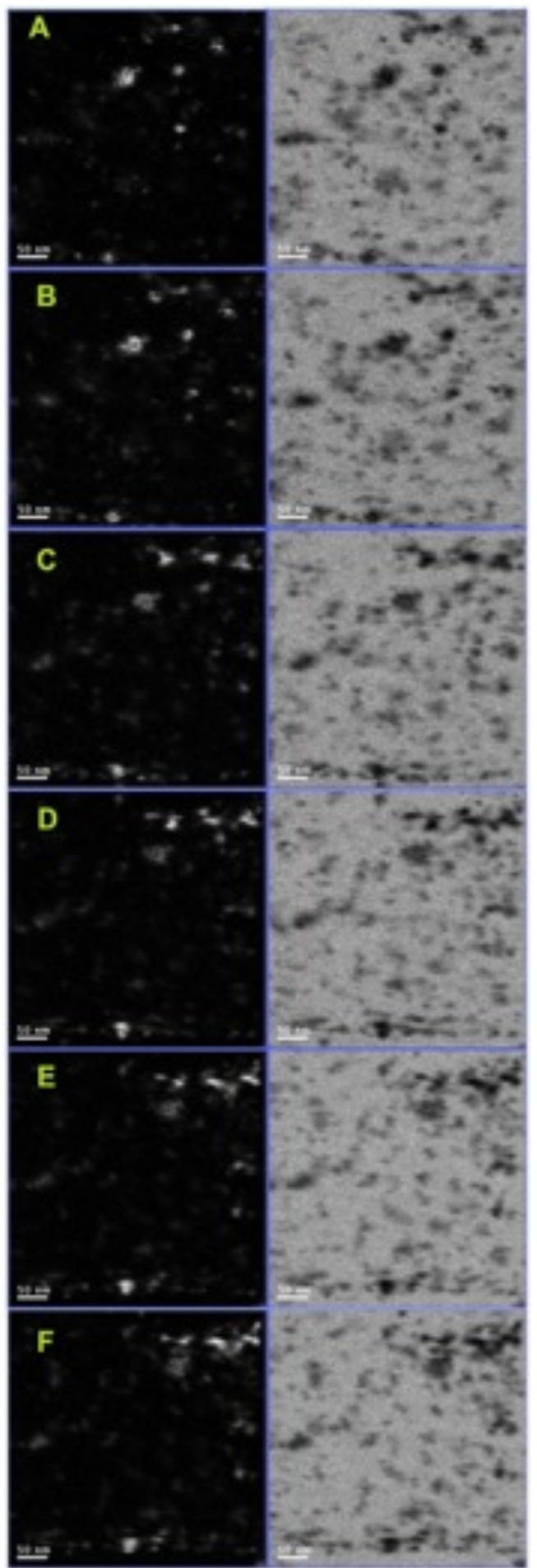

Figure 2: STEM images (DF image left, BF image right, mag $\mathrm{x} 400 \mathrm{k}$ ) over time during e-beam growth of Pd particles within the surfactant support. Each image is $\sim 25$ seconds after the proceeding image. In the upper right corner of the images, a set of small Pd particles can be seen moving towards each other (A-D), and agglomerating to form a larger Pd network (E, F). 